\title{
Review on Design of Control Systems for Tethered UUVs using PID Controllers
}

\author{
Ms. Devjani Bhattacharya \\ Assistant Professor \\ Department of Electrical and Electronics \\ Dayananda Sagar College of Engineering \\ Bengaluru, India
}

\author{
Dr. H L Viswanath \\ Professor \\ Department of Electrical \& Communication \\ Engineering, Dayananda Sagar College of Engineering \\ Bengaluru, India
}

\begin{abstract}
Unmanned Underwater Vehicles have gained popularity for the last decades, especially for the purpose of not risking human life in dangerous operations. On the other hand, underwater environment introduces numerous challenges in control, navigation and communication of such vehicles.Certainly, this fact makes the development of these vehicles more interesting and engineering-wise more attractive.
\end{abstract}

Conventional Proportional-Integral-Derivative (PID) controllers exhibit moderately good performance once the PID gains are properly tuned. However, when the dynamic characteristics of the system are time dependent or the operating conditions of the system vary, it is necessary to retune the gains to obtain desired performance. Self-tuning of PID controllers has emerged as a new and active area of research with the advent and easy availability of algorithms and computers. The proposed study

operated and autonomous underwater vehicles. The difference between an autonomous underwater vehicle, or AUV, and a ROV
Keywords: UUV, ROV, Control-Navigationcommunication, Taguchi's method, self tuning PID controllers.

\section{I.INTRODUCTION}

The term UUV is a generic expression to describe both remotely operated and autonomous underwater vehicles. between an autonomous underwater vehicle, or AUV is that the ROV is connected to a command platform (for instance a ship) with a tethered cable or an acoustic link [3]. The tethered cable ensures energy supply and information signals, in this way an operator is able to constantly monitor and control the vehicle. The AUV however, is equipped with a battery pack and sonar to fulfill its mission avoiding the use of an operator, which introduces conflicting control requirements. It has to be simple enough to ensure an online implementation of control techniques but at the same time has to cope with a time-varying vehicle/environment interaction. 


\section{II.UUV SPECIFICATION}

In the proposed study, an experimental remotely operated vehicle as a test platform for experiments and analysis is considered [2]. This is a torpedoshaped under actuated ROV, without any side thrusters to control the sway direction (this is not implemented because of economical and weight considerations) (Fig. 1).

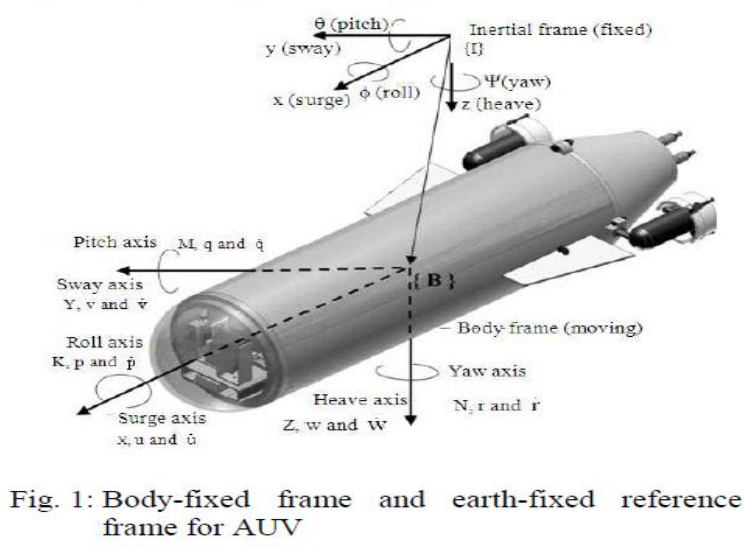

There are only two stern propellers which are offering control inputs as the force in the surge direction and the control torque in yaw direction in the horizontal plane. Commands as well as the tracking error are used in arriving at the optimal gains.

\section{Problem definition}

In order to achieve a high degree of autonomy, a suitable controlling method of underwater vehicles is very challenging due to the nature of underwater dynamics and parameter uncertainties (disturbances, wind, waves, ocean-currents etc.).In this proposed work, among the mentioned problems, control of underwater vehicles, particularly the motion control will be focused upon.

\section{Methodology}

This section describes the methodology used in the proposed work. The problem specification will be identified first to have a clear view in order to overcome the limitations, weakness and to improve the systems. For the Unmanned Underwater Vehicle, the most crucial issue is the control system needed by the ROV to perform the underwater applications and tasks.

\section{Control mechanism of UUV}

The control scheme will be tested in simulation studies using a six degrees of freedom UUV model which contains both kinematic and dynamic elements.

\section{Motion control}

The motion of UUVs can be described in 6 degreesof freedom (DOF), since 6 independent coordinates are necessary to determine the position and orientation of a rigid body. The 6 different motion components are defined as 'surge','sway', 'heave', 'roll', 'pitch' and 'yaw', as shown in Table 1 [1].

Table 1. Notation used for the marine vehicles

\begin{tabular}{|c|c|c|c|c|}
\hline$\stackrel{1}{0}$ & & 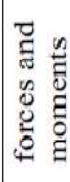 & 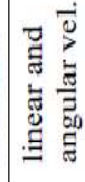 & 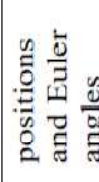 \\
\hline 1 & motions in the $x$-direction (surge) & $X$ & $u$ & $x$ \\
\hline 2 & motions in the $y$-direction (sway) & $Y$ & $v$ & $y$ \\
\hline 3 & motions in the $z$-direction (heave) & Z & $w$ & $z$ \\
\hline 4 & rotation about $x$-axis (roll) & K & $p$ & $\phi$ \\
\hline 5 & rotation about $y$-axis (pitch) & $M$ & $q$ & $\theta$ \\
\hline 6 & rotation about $z$-axis (yaw) & $N$ & $r$ & $\psi$ \\
\hline
\end{tabular}


When analyzing the motion of marine vehicles in 6 DOF, it is convenient to define two coordinate frames as indicated in Fig. 2. The moving coordinate frame $X 0 Y 0 Z 0$ is fixed to the

vehicle and referred to as 'the body-fixed reference frame'. The origin $O$ of the body-fixed frame is usually chosen to coincide with the 'center of gravity (CG)', when $\mathrm{CG}$ is in the principal plane of symmetry or at any other convenient point if this is not the case.

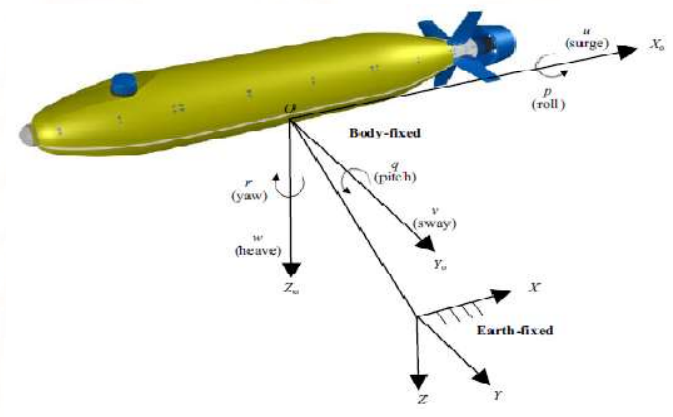

Fig. 2. Body-fixed and earth-fixed reference frames

\section{Self-tuning of PID controller using Taguchi's} method:

The proposed controller scheme for the UUV is shown in Fig. 3. The trajectory controller generates the desired trajectory from the user inputs. User inputs consist of start point, goal point, way points and vehicle speed or time duration. These desired values are compared with the actual values which are coming from the ROV dynamic model (sensor values in the realtime). Comparator is giving tracking errors and these error values are fed into the PID controller and controller is generating necessary control signals, as per the control law. The self-tuning block calculates the optimal values of the controller gains to reduce the tracking errors. The real-time calculations of gains can be achieved by implementing the Taguchi's robust optimization method. The variations in the input commands as well as the tracking error are used in arriving at the optimal gains [2].

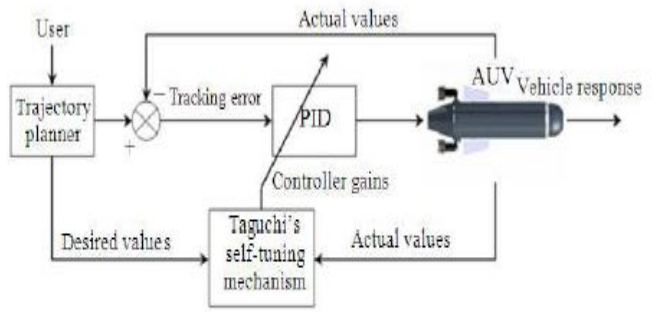

Fig. 3: Block diagram of proposed self tuning controller structure

\section{PID controller methodology}

Using PID controller tracking control of underwater vehicle is proposed. Self-tuning of PID controllers has emerged as a new and active area of research and development with the advent and easy availability of algorithms and computers [2].

Self-tuning mechanism will avoid time consuming manual tuning of controllers and promises better results by providing optimal PID controller settings as the system dynamics or operating points change. Most of the self-tuning methods available are based on frequency response characteristics and search methods. In this study, we proposed a method based on Taguchi's robust design method for self-tuning of an autonomous underwater vehicle controller. The algorithm, based on this method, will tune the controller gains optimally and robustly in real time with less computation effort by using desired and actual state variables.

It can be used for the Single-Input Single-Output (SISO) systems as well as Multi-Input Multi-Output 
(MIMO) systems without mathematical models of plants.

\section{Stability of Underwater Vehicles}

Stability of an underwater vehicle can be defined as "the ability of returning to an equilibrium state of motion after a disturbance without any corrective action, such as use of thrusters power or control surfaces"[1]. Hence, maneuverability can be defined as the capability of the vehicle to carry out specific manoeuvres.

At this point, the following issue about the stability shall be emphasized. Excessive stability implies very high control effort; whereas it would be easy to control a marginally stable vehicle.

Consequently, there exists a compromise between

\section{Stability of Underwater Vehicles}

Stability of an underwater vehicle can be defined as "the ability of returning to an equilibrium state of motion after a disturbance without any corrective action, such as use of thrusters power or control surfaces"[1]. Hence, maneuverability can be defined as the capability of the vehicle to carry out specific manoeuvres.

At this point, the following issue about the stability shall be emphasized. Excessive stability implies very high control effort; whereas it would be easy to control a marginally stable vehicle.

Consequently, there exists a compromise between stability and maneuverability.Furthermore, it makes sense to distinguish between controls-fixed (open- loop) and controls-free (closed-loop) stability. The essential difference between these terms can be stated as follows:

- Open-loop stability implies investigating the vehicle's stability when the control surfaces are fixed, and when the thrust from all the thrusters is constant.

- Closed-loop stability refers to the case when both the control surfaces and the thruster power are allowed to vary. This implies that the dynamics of the control system must also be considered in the stability analysis.

\section{Communication}

The ROV system is unmanned, highly maneuverable and it is operated by a person on board of a vessel. It will be linked to the ship by a tether (optical fibre cables).

As the sophistication of acoustic sensor and communication systems related to unmanned underwater vehicles (UUV) has increased, the requirement for greater volume and higher speed data transfers has emerged. Fiber optic technology provides an effective means for high bandwidth communications with a UUV while minimizing weight and space criteria aboard the UUV [3]. Increase in data transmission speed has permitted real time processing of data on the launch platform when using large high powered computing systems. Maximum system reliability at advanced performance levels can also be realized.

Modern cables are typically about 25 millimetres (0.98 in) in diameter and weigh around 1.4 kilograms per metre $(0.4 \mathrm{lb} / \mathrm{ft})$ for the deep-sea sections which 
comprise the majority of the run, although larger and heavier cables are used for shallow-water sections near shore.

\section{CONCLUSION}

The considered experimental ROV will be hosted in the MATLAB/Simulink environment.

Simulation results show the viability and attractiveness of the approach adopted.

Each underwater vehicle should have a motion control system specific to its characteristics and needs.

Although, numerous control strategies which were successfully applied for the motion control problem of underwater vehicles exist and are literally accurate, it is hard to determine which approach is the most suitable and furthermore applicable to our cases. Not only for the motion, but also for the mission and formation control, the most optimal algorithms should be adopted and strategies should be carefully chosen in order to acquire robust underwater vehicles that will perform critical applications.

Regarding motion control of underwater vehicles, utilization of more advanced system control systems is inevitable in order to design more intelligent, adaptive, and robust controllers that provide optimal control solution in terms of non-linearity handling, and cost minimization.

\section{References}

[1] Özgür YILDIZ, R. Bülent GÖKALP, and A. Egemen YILMAZ,

"A Review on Motion Control of the Underwater Vehicles".

https://www.researchgate.net/publication/224091430_ A_review_on_motion_control_of_the_Underwater_V ehicles, 2009 International Conference on Electrical and Electronics Engineering (ELECO 2009) Bursa, Turkey 5-8 November 2009, IEEE Catalog Number: ISBN: CFP0918H-PRT 978-1-4244-5106-7

[2] A Self-Tuning Proportional-Integral-Derivative Controller for an Autonomous

Underwater Vehicle, Based On Taguchi Method, M. Santhakumar and T. Asokan

Department of Engineering Design, Indian Institute of Technology Madras, Chennai (600036), India, Journal of Computer Science 6 (8): 862-871, 2010,ISSN 1549-3636, (C) 2010 Science Publications

[3] An integrated, underwater optical /acoustic communications system, N. Farr, A. Bowen, J. Ware, C. Pontbriand, Applied Ocean Physics and Engineering, M. Tivey, Geology and Geophysics, Woods Hole Oceanographic Institution, Woods Hole, MA 02543 USA

[4] FUNDAMENTALS OF PHOTONICS, Fibre optic telecommunication, Nick Massa, Springfield Technical Community College, Springfield, Massachusetts. Massa, Nick. "Fiber optic telecommunication." Fundamentals of Photonics. University of Conneticut (2000). 
[5] T. I. Fossen, Guidance and Control of Ocean

Vehicles, John Wiley \& Sons Ltd, ISBN: 0-471-

94113-1, 1994.

[6] H. Mahesh, J. Yuh and R. Lakshmi, “A

Coordinated Control of an Underwater Vehicle and

Robotic Manipulator", Journal of Robotic Systems, vol. 8, no. 3, pp. 339-370, 1991.

[7] Antonelli, G., 2007. On the use of adaptive/integral actions for six-degrees-of-freedom control of autonomous underwater vehicles. IEEE J.

Ocean. Eng., 32: 300-312.

DOI:10.1109/JOE.2007.893685

[8] J. Yuh and K. V. Gonugunta, "Learning Control of Underwater Robotic Vehicles", Proc. IEEE Int'l Conf. On Robotics and Automation, vol.2, pp. 106-111, 1993.

[9] Astrom, K.J. and T. Hagglund, 1988. Automatic Tuning of PID Controllers. 1st Edn., Instrumentation Systems, Research Triangle Park, ISBN: 13:9781556175169, pp: 141 .

[10] M. Caccia, G. Veruggio, "Guidance and control of a reconfigurable unmanned underwater vehicle", Control Engineering Practice, vol. 8, no. 1, pp. 21-37, January 2000 